\title{
Generation and Growth of Atomic-scale Roughness at Surface and Interface of Silicon Dioxide Thermally Grown on Atomically Flat Si Surface
}

\author{
Yuusuke Hayashi ${ }^{\mathrm{a}}$, Ryu Hasunuma ${ }^{\mathrm{b}}$ and Kikuo Yamabe ${ }^{\mathrm{c}}$ \\ Institute of Applied Physics, University of Tsukuba \\ 1-1-1 Tennoudai, Tsukuba, Ibaraki, 305-8573 Japan \\ aemail: beppo@edu.esys.tsukuba.ac.jp, bemail: hasunuma@bk.tsukuba.ac.jp \\ cemail: yamabe@esys.tsukuba.ac.jp,
}

Keywords: Silicon, Oxidation, Uniformity, Roughness,

\begin{abstract}
Atomic force microscopy measurements are carried out on the surface and interface of $\mathrm{SiO}_{2}$ thermally grown on an atomically flat $\mathrm{Si}$ surface, and the uniformity of thickness on an atomic scale is investigated. Protuberances on the surface of $\mathrm{SiO}_{2}$ grown at $800{ }^{\circ} \mathrm{C}$ in $3.8 \% \mathrm{O}_{2}$ diluted by argon at the atmospheric pressure are generated in the initial stage of oxidation and subsequently increases in height at the same positions. The surface RMS value of $\mathrm{SiO}_{2}$ linearly increases up to the thickness of approximately $5 \mathrm{~nm}$, which almost corresponds to what was called the initial oxidation thickness. This fact indicates that the roughness increase is related to the re-oxidation of $\mathrm{SiO}$ emitted from the $\mathrm{Si} / \mathrm{SiO}_{2}$ interface during the initial oxidation. Additionally, the surface RMS value is saturated at a value of approximately $0.13 \mathrm{~nm}$. A correspondence between the position of the dimples on the surface and the positions of the protuberances at the interface is also clear. The fact is the direct evidence of the local thinning of the thermally grown $\mathrm{SiO}_{2}$ films. Based on these results, an atomic scale thermal oxidation mechanism is discussed.
\end{abstract}

\section{Introduction}

A non-uniform gate dielectric film can induce a fluctuation in the threshold voltages or lifetimes of time-dependent dielectric breakdown of ultra-fine metal-insulator-semiconductor field-effecttransistors (MISFET's). It is very important to clarify the underlying generation mechanism of such thickness variatiopns. On the other hand, dielectric breakdown is also a local phenomenon: the dielectric film breaks down at its weakest point. To realize the higher reliability of the dielectric films, a more uniform film thickness on an atomic-scale is needed. For example, for the dielectric films with a thickness of approximately $3 \mathrm{~nm}$, a variation of a few atomic layers is equivalent to approximately $10 \%$ of the total film thickness. Such film thickness variation also induces a fluctuation in the direct tunnel leakage current [1].

The Deal-Grove model is well known for thermal oxidation of silicon [2]. In this model, the oxidizing-species diffuse across a preexisting oxide layer, and react at the silicon surface, and a new 
$\mathrm{SiO}_{2}$ is formed there. A volume enhancement of 2.3 times at the interface induces mechanical stress that affects various aspects of thermal oxidation.

On the other hand, it has been reported that thermal oxidation of an atomically flat $\mathrm{Si}$ under lower oxygen pressure progresses in a layer-by-layer manner in a $\mathrm{SiO}_{2}$ film thickness of a few atomic layers [3]. If the layer-by-layer oxidation is strictly maintained, a similar topography should be produced at the $\mathrm{Si} / \mathrm{SiO}_{2}$ interface during thermal oxidation. A precise evaluation of the oxidation process has shown that although thermal oxidation at the atmospheric pressure can be thought of as a layer-by-layer process, strictly speaking, it proceeds in a

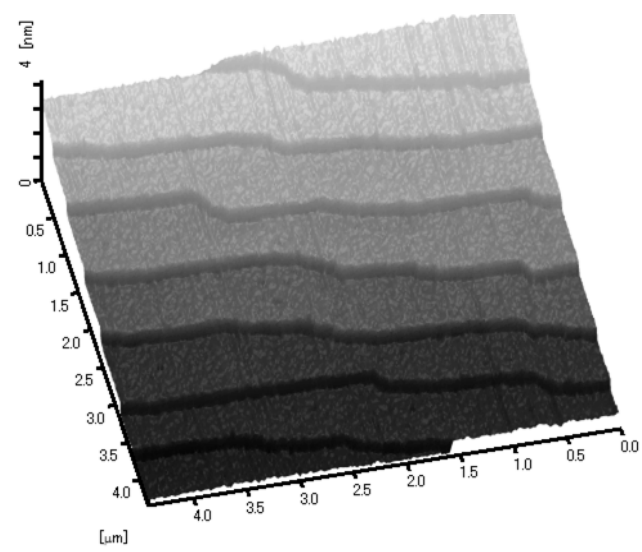

Fig. 1 Well-defined step/terrace structure on a (111)-oriented Si surface after LOW treatment. The typical RMS value within a terrace surface is less than 0.05 $\mathrm{nm}$. multilayer manner $[4,5]$. Neither the roughening mechanism nor the thickness uniformity during thermal oxidation at the atmospheric pressure has so far been clarified.

In the present study, both issues were investigate by precise observations of the topography at the $\mathrm{SiO}_{2}$ surface and $\mathrm{Si} / \mathrm{SiO}_{2}$ interface using atomic force microscopy (AFM).

\section{Experimental}

Czochralski-grown (111)-oriented mirror-polished Si wafers were used. After modified RCA cleaning, the wafers were immersed in ultralow dissolved-oxygen water (LOW). The typical root mean square (RMS) value at the surface of a commercial-base mirror-polished silicon wafer is more than $0.13 \mathrm{~nm}$. After LOW immersion, the well-defined surface covered with single atomic step lines and atomically flat terraces were formed as shown in Fig. 1 [6, 7]. After LOW treatment, a Si wafers were thermally oxidized in $3.8 \% \mathrm{O}_{2}$ diluted with argon at 800 and $1000{ }^{\circ} \mathrm{C}$. After the thermal oxidation, the topography of the $\mathrm{SiO}_{2}$ surfaces was observed using AFM. The $\mathrm{Si} / \mathrm{SiO}_{2}$ interface topography was observed after removal of $\mathrm{SiO}_{2}$ films. The RMS value within a 200 x 200 $\mathrm{nm}^{2}$ area was used as an indicator of the roughness. On (111)-oriented Si wafers, such well-defined step/terrace structures can be easily obtained. $\mathrm{The}_{\mathrm{SiO}}$ films were removed to observe the $\mathrm{Si} / \mathrm{SiO}_{2}$ interface structure. The $\mathrm{Si}(111)$ surface was found to be sufficiently stable against attack by this solution, but the $\mathrm{Si}(100)$ surface was not. Therefore, the (111)-oriented wafers were used in this work.

\section{Results and Discussion}

Figure 2 shows typical AFM images of the surface and interface of 2.1-, 5.4-, and $12.6 \mathrm{~nm}$-thick $\mathrm{SiO}_{2}$ grown at $800{ }^{\circ} \mathrm{C}$ in $3.8 \% \mathrm{O}_{2}$ diluted with argon. Profiles along the solid lines in each image are also shown at the same height scale of $0.75 \mathrm{~nm}$. It is seen that with increasing film thickness, the roughness at both the surface and interface increases. Figure 3(a) shows the $\mathrm{SiO}_{2}$ film thickness 

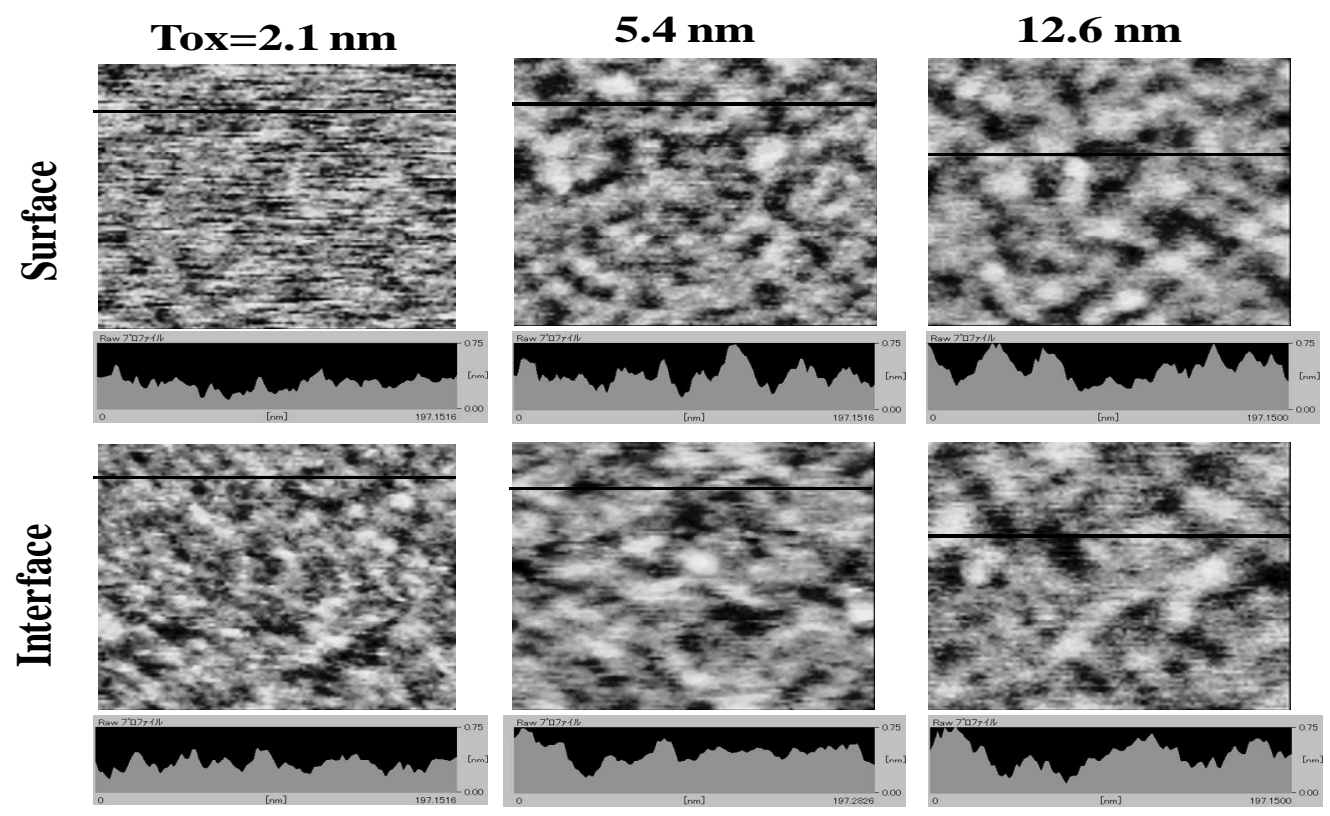

Fig. 2 Typical AFM images of the surface and interface of 2.1-, 5.4-, and $12.6 \mathrm{~nm}$ thick $\mathrm{SiO}_{2}$ films thermally grown at $800{ }^{\circ} \mathrm{C}$ in $3.8 \% \mathrm{O}_{2}$ diluted with argon.

roughness at both the surface and interface increases. Figure 3(a) shows the $\mathrm{SiO}_{2}$ film thickness dependence of the RMS value at $\mathrm{SiO}_{2}$ surface. The typical RMS value of an initial atomically flat $\mathrm{Si}$ terrace before thermal oxidation was less than $0.05 \mathrm{~nm}$. With increasing film thickness, the RMS value increased almost linearly for thicknesses of less than $5 \mathrm{~nm}$ and saturated at a film thickness of approximately $5 \mathrm{~nm}$. The saturation thickness for oxidation at $800{ }^{\circ} \mathrm{C}$ is more than that at $600{ }^{\circ} \mathrm{C}$ and is less than that at $1000{ }^{\circ} \mathrm{C}$ [8]. That is, as the oxidation temperature increased, the roughening rate decreased, and the saturation thickness increases. In Fig. 3(b), the RMS value of the interface is plotted as a function of the film thickness. Although the data is scattered, the RMS value of the interface is also saturated at approximately $5 \mathrm{~nm}$.
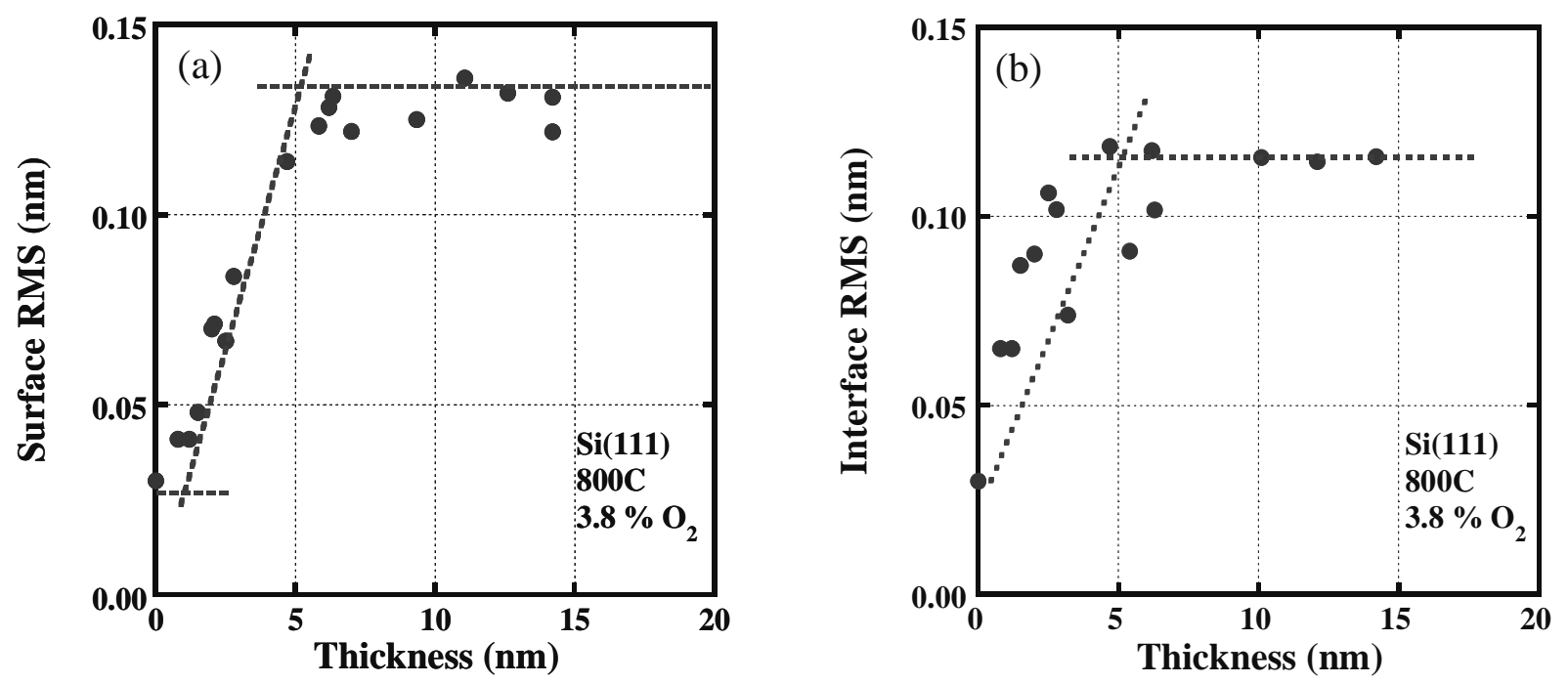

Fig. 3 (a) Surface RMS value and (b) interface RMS value plots as a function of thickness of the $\mathrm{SiO}_{2}$ films grown at $800{ }^{\circ} \mathrm{C}$ in $3.8 \% \mathrm{O}_{2}$ diluted with argon. 
In Fig. 4, the relation between film thickness and oxidation time is plotted in the case for thermal oxidation at $800{ }^{\circ} \mathrm{C}$ in $3.8 \% \mathrm{O}_{2}$ ambient. The thickness of $\mathrm{SiO}_{2}$ thermally grown in the initial oxidation region is approximately $4 \mathrm{~nm}$, which is almost the same as the saturation thickness described above. The initial oxidation thickness at $800{ }^{\circ} \mathrm{C}$ is less than that at $1000{ }^{\circ} \mathrm{C}$. It is presumed that this is related to the diffusion length of the $\mathrm{SiO}$ emitted from the $\mathrm{Si} / \mathrm{SiO}_{2}$ interface $[9,10]$ to relax the oxidation stress. The steep increase of film thickness in the initial oxidation region was simulated taking into account the re-oxidation of the emitted $\mathrm{SiO}$. The correlation between the roughness saturation

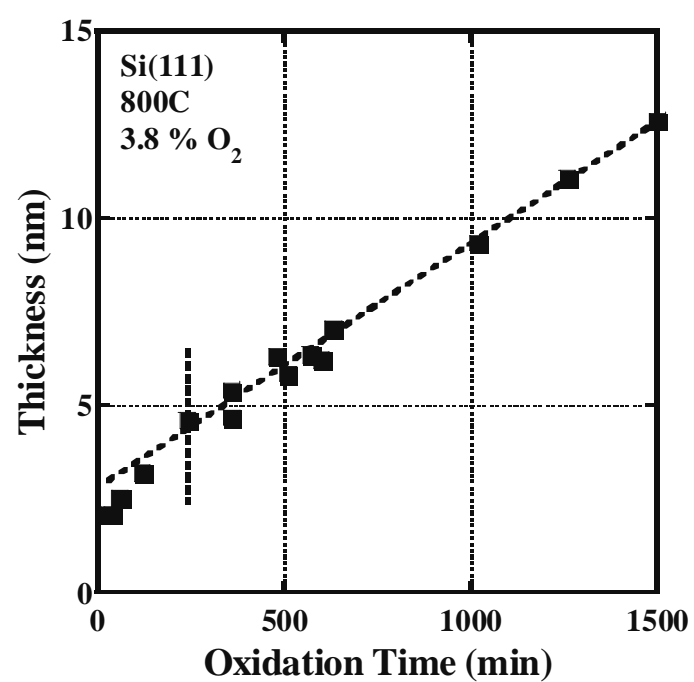

Fig. $4 \mathrm{SiO}_{2}$ film thickness as a function of oxidation time at $800{ }^{\circ} \mathrm{C}$ in $3.8 \% \mathrm{O}_{2}$. diluted by argon. thickness and the initial oxidation thickness indicated that the generation and growth of the roughness is closely related to the re-oxidation of the $\mathrm{SiO}$ emitted from the $\mathrm{Si} / \mathrm{SiO}$ interface[11].

In the LOW treatment, an $\mathrm{SiO}_{2}$ on the $\mathrm{Si}$ surface is not attacked [12]. We formed a step/terrace structure within the $\mathrm{Si}$ surface surrounded by a thick $\mathrm{SiO}_{2}$ film using photo-lithography. The $\mathrm{SiO}_{2}$ pattern was used as a guide to observe the surface morphology at the same position on the $\mathrm{SiO}_{2}$ film thermally grown on the Si terrace.

Figure 5 shows typical surface AFM images of four thicknesses of $\mathrm{SiO}_{2}$ thermally grown on an atomically flat silicon terrace. By using the $\mathrm{SiO}_{2}$ patterns and step line patterns as two guides, we can recognize that ongoing thermal oxidation changes the surface morphology of the $\mathrm{SiO}_{2}$ in the same region. When the formed $\mathrm{SiO}_{2}$ is thin, the step line patterns are taken over from the initial silicon surface to the $\mathrm{SiO}_{2}$ surface. A number of the protuberances are generated just after oxidation starts. Subsequently, the height of the protuberances is increased by oxidation, with almost no change in their locations.
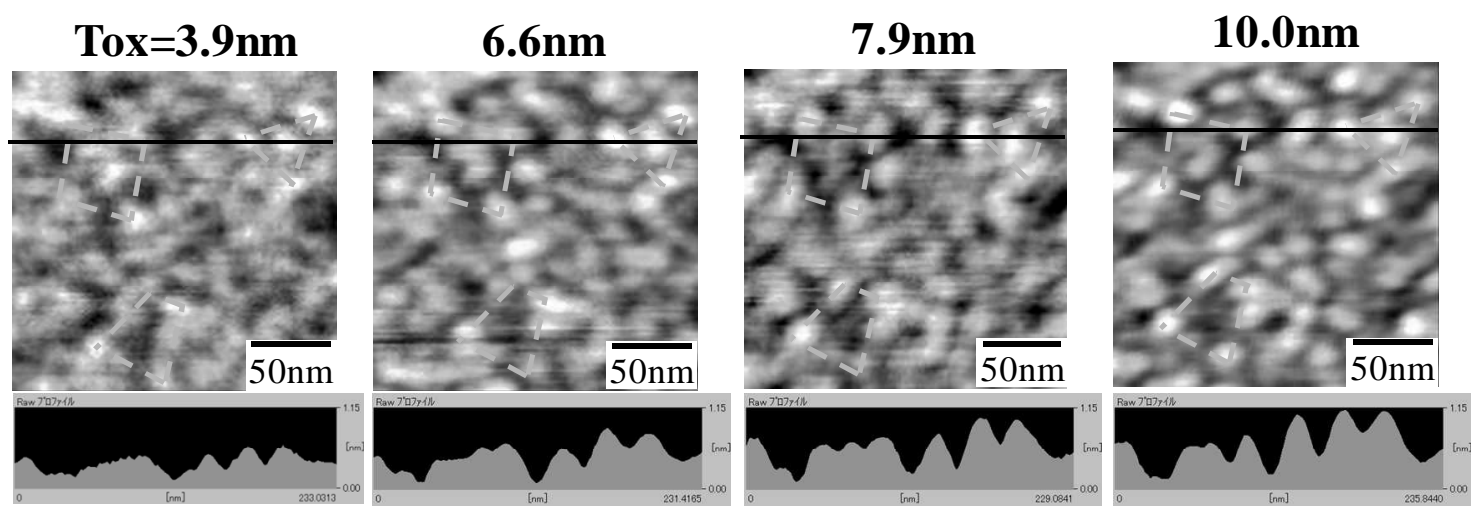

Fig. 5 AFM images of the surfaces of $\mathrm{SiO}_{2}$ grown at $1000{ }^{\circ} \mathrm{C}$ in $3.8 \% \mathrm{O}_{2}$ ambient. The film thicknesses are 3.9-, 6.6-, 7.9-, and 10.0-nm. The triangle and quadrangles in each figure indicate the same set of the protuberances. 


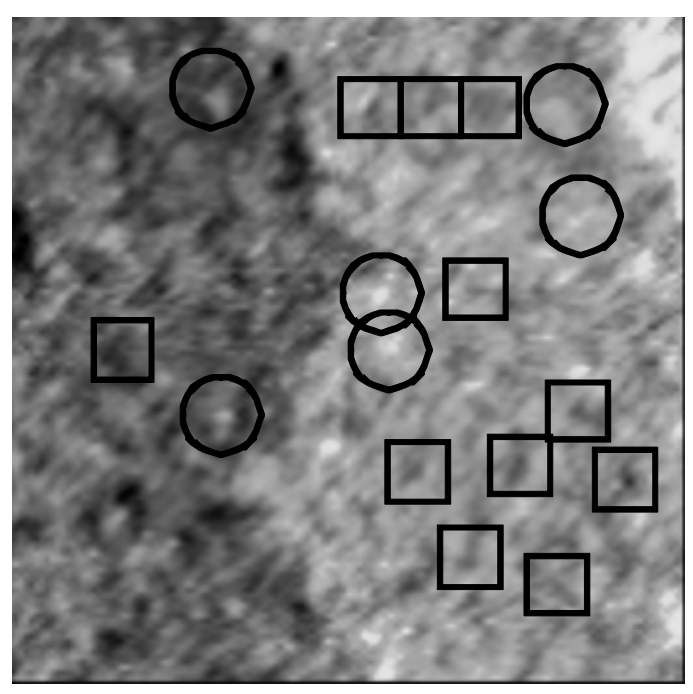

(a) Surface

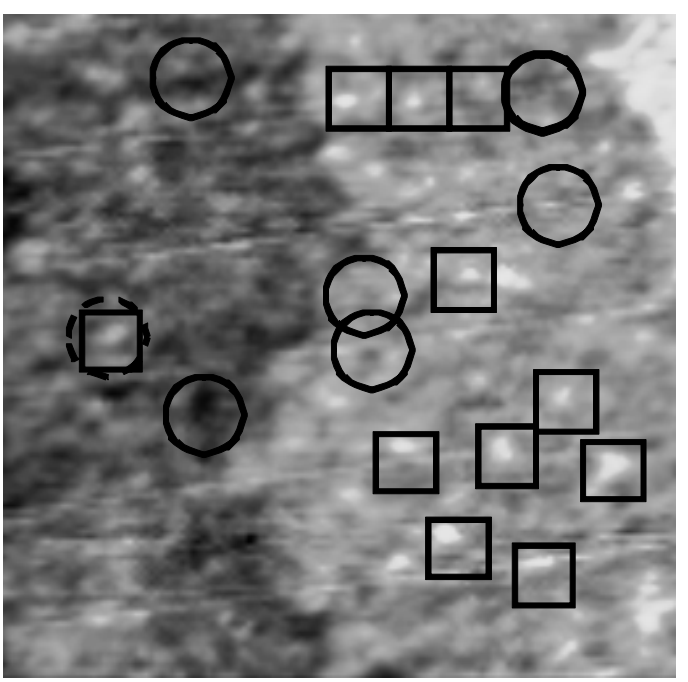

(b) Interface

Fig. 6 Typical AFM images of the surface and interface in the same area of $500 \mathrm{~nm} \times 500 \mathrm{~nm}$ square area. Circles and squares on the corresponding positions at the surface and interface are drawn.

Using a technique wherein surface topographies of $\mathrm{SiO}_{2}$ with various film thicknesses can be observed in the same region, the two-dimensional correlation of topography between the $\mathrm{SiO}_{2}$ surface and $\mathrm{Si} / \mathrm{SiO}_{2}$ interface needs to be investigated to clarify local thinning of thermally grown $\mathrm{SiO}_{2}$ films. In Figs. 6(a) and 6(b), the typical AFM images of the surface and interface of $\mathrm{SiO}_{2}$ with a thickness of $5.6 \mathrm{~nm}$ are shown. Based on the zigzag step line pattern in this figure, it is confirmed that the images are of the surface and interface of the $\mathrm{SiO}_{2}$ in the same region. The circles and squares in both images are drawn the corresponding locations. The circles and squares in Fig. 6(a) indicate the positions of protuberances and dimples on the $\mathrm{SiO}_{2}$ surface image, respectively. It can be noticed that in these positions the protuberances on the $\mathrm{SiO}_{2}$ surface correspond to the dimples at the $\mathrm{Si} / \mathrm{SiO}_{2}$ interface and vice versa. Since it is confirmed that the immersion in diluted hydrofluoric acid (d-HF) maintains the atomic structure of the $\mathrm{Si}$ surface, the dimples appear after removal of the formed $\mathrm{SiO}_{2}$ by d-HF immersion. At the $\mathrm{Si} / \mathrm{SiO}_{2}$ interface, the dimples indicate the occurrence of local oxidation. The correspondence between the position of the protuberances at the surface and the dimples at the interface indicates is one of

$\uparrow \quad$ SiO emission $\checkmark$ Push-up effect Protuberances

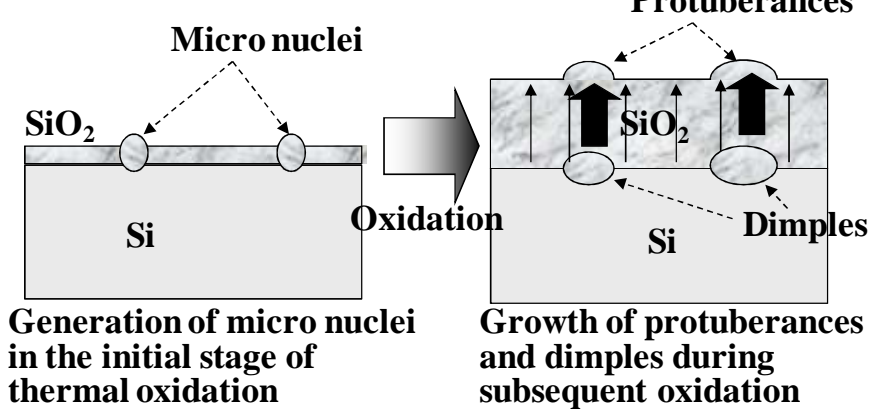

Fig. 7 A model of nonuniform oxidation. Two mechanisms of the $\mathrm{SiO}$ emission from the $\mathrm{Si} / \mathrm{SiO}_{2}$ interface and the push-up of the $\mathrm{SiO}_{2}$ by the local oxidation at the interface contribute to the protuberance growth at the $\mathrm{SiO}_{2}$.surface. origins of the protuberances at the $\mathrm{SiO}_{2}$ 
surface. As shown in Fig.7, micro nuclei which are the origins of the protuberances at the surface and the dimples at the interface are generated immediately after the start of the oxidation. The preexisting thin $\mathrm{SiO}_{2}$ film is then pushed up by the oxide growing locally growing at the interface. In other words, the correspondence in Fig. 6 indicates that the position of local oxidation by the multilayer oxidation is determined at the initial stage of the oxidation. During subsequent oxidation, the position of the preceding local oxidation is maintained. However, the push-up effect alone cannot explain the saturation of the surface RMS value shown in Fig.3. Re-oxidation of the $\mathrm{SiO}$ emitted from the interface should also be taken into account. A comparison of Fig. 3 described above and Figs. 3 and 6 in Ref. 11 suggests that the relative contribution of each mechanism depends on the oxidation condition used.

On the other hand, the correspondence of the dimples at the surface and the protuberances at the interface indicates that the oxidation is locally delayed there. That is, the local thinning of the $\mathrm{SiO}_{2}$ film occurs. This influences the dielectric breakdown characteristics of the $\mathrm{SiO}_{2}$ films.

\section{Summary}

Precise AFM observations of the surface and interface of $\mathrm{SiO}_{2}$ thermally grown at $800{ }^{\circ} \mathrm{C}$ in $3.8 \%$ $\mathrm{O}_{2}$ diluted with argon at the atmospheric pressure on atomically flat $\mathrm{Si}$ surface have been carried out to determine the $\mathrm{SiO}_{2}$ thickness uniformity on an atomic scale. Protuberances generated at the initial stage of oxidation were found to increase in height as oxidation proceeds. The surface RMS value of $\mathrm{SiO}_{2}$ was found to increase linearly up to a thickness of approximately $5 \mathrm{~nm}$, which almost corresponds to the thickness of the initial oxidation layer. This fact indicates that the roughness increase is related to re-oxidation of $\mathrm{SiO}$ emitted from the $\mathrm{Si} / \mathrm{SiO}_{2}$ interface during the initial oxidation. In addition, the surface RMS value saturated at an RMS value of approximately 0.13 $\mathrm{nm}$. A correspondence between the position of dimples at the surface and the positions of the protuberances at the interface was also observed. This is the direct evidence of the local thinning of the thermally grown $\mathrm{SiO}_{2}$ films. These experimental results were clarified for a wide atomically flat silicon terrace after LOW immersion.

\section{Acknowledgement}

This work was partially supported by the Ministery of Education, Culture, Sports Science and Technology of Japan (No. 19026002A020001, 20035002) and the Ministry of Economy, Trade and Industry of Japan.

\section{References}

[1] R. Hasunuma, J. Okamoto, N. Tokuda and K. Yamabe: Jpn.J.Appl.Phys., Vol. 43 (2004), p.7861.

[2] B. E. Deal and A. S. Grove: J.Appl.Phys., Vol. 36 (1965), p.3770.

[3] H.Watanabe and M.Ichikawa: Phys.Rev.B, Vol. 55 (1997), p.9699. 
[4] D.Hojo, N.Tokuda and K.Yamabe: Jpn.J.Appl.Phys., Vol. 41 (2002), p.L505.

[5] D.Hojo, H.Oeda, N.Tokuda and K.Yamabe: Jpn.J.Appl.Phys., Vol. 42 (2003), p.1903.

[6] N.Tokuda, D.Hojo, S.Yamasaki, K.Miki and K.Yamabe: Jpn.J.Appl.Phys., Vol. 42 (2003), p. L1210.

[7] N.Tokuda, M.Nishizawa, K.Miki, S.Yamasaki, R.Hasunuma and K.Yamabe: Jpn.J.Appl.Phys., Vol. 44 (2005), p.L613.

[8] R. Hasunuma, Y.Hayashi and K. Yamabe: in preparation.

[9] H. Kageshima, K.Shiraishi and M.Uematsu, Jpn.J.Appl.Phys., Vol. 38 (1999), p.L971.

[10] M.Uematsu, H.Kageshima and K.Shiraishi: Jpn.J.Appl.Phys., Vol. 39(2000), p.L1135.

[11] K.Yamabe, K.Ohsawa, Y.Hayashi and R.Hasunuma: J.Electrochem.Soc., Vol. 156 (2009) p.G201.

[12] D. Hojo, N. Tokuda and K. Yamabe: Jpn.J.Appl.Phys., Vol. 42. Part 2 (2003), p. L561. 